\title{
Ökonomen, Publikationen und Zitationen: Ein europäischer Vergleich
}

\author{
Reiner Eichenberger* \\ Universität Freiburg \\ Ursina Meier** \\ Universität Bern
}

und Rolf Arpagaus $\ddagger$

Universität Freiburg

\section{$\overline{\text { I. Einleitung }}$}

Die moderne Ökonomik scheint eine angelsächsische Wissenschaft zu sein. Diesen Eindruck vermitteln die Reihe der Wirtschaftswissenschafts-Nobelpreisträger genau so wie quantitative Analysen der Autoren- und Herausgeberschaft führender wirtschaftswissenschaftlicher Zeitschriften (vgl. z.B. Hodgson und Rothman 1999, Kalaitzidakis, Mamuneas und Stengos 1999). Die gleiche Vermutung nährt auch das „Who's Who in Economics“ von Blaug (1986, 1999): ${ }^{1}$ Von den 1985 lebenden, meistzitierten 877 Ökonomen arbeiteten 594 in den USA und 122 in Grossbritannien, aber nur gerade 20 in Deutschland und 26 in Frankreich. Genau so wie die Amerikaner weltweit führend sind, sind es die Briten in Europa. Bemerkenswert ist, dass der Einfluss der europäischen Ökonomen im Laufe des 20. Jahrhunderts stark abgenommen hat, was v.a. auf die Deutschen, Franzosen und Italiener, jedoch weniger auf die Briten zutrifft (dazu ausführlich Frey und Pommerehne, 1988 und Pommerehne und Frey, 1988).

\footnotetext{
* Seminar für Finanzwissenschaft, Universität Freiburg, Av. de Beauregard 13, CH-1700 Freiburg. Tel. +41026300 82 65, E-Mail: Reiner.Eichenberger@unifr.ch

** Volkswirtschaftliches Institut, Universität Bern

$\ddagger$ Wir danken Bruno Frey, Hermann Garbers und Margit Osterloh für hilfreiche und wertvolle Hinweise.

1. Die Ende 1999 erschienene dritte Auflage des „Who's Who in Economics” konnte für diesen Aufsatz noch nicht umfassend ausgewertet werden, da es erst nach der Fertigstellung des Manuskripts dieses Aufsatzes verfügbar wurde. Wie eine erste Auswertung jedoch zeigt, behalten die hier gemachten Aussagen volle Gültigkeit.
} 


\section{Reiner Eichenberger, Ursina Meier und Rolf Arpagaus}

Diese Beobachtungen können auf verschiedene Weise zu erklären versucht werden. Die unterschiedliche Struktur der akademischen Märkte sowie der Institutionen für Forschung und Lehre (z.B. Frey und Eichenberger 1993), unterschiedliche Beherrschung der englischen Sprache und allgemeine gesellschaftliche Umwälzungen infolge des 2. Weltkriegs sind einige mögliche Ursachen. Weil die Ursachen vielfältiger Natur sind, ist auch die zukünftige Entwicklung des Einflusses der kontinental-europäischen Ökonomen ungewiss. Sprachbarrieren dürften unwichtiger werden, und Kontinentaleuropa sollte sich irgendwann auch wissenschaftlich vom 2. Weltkrieg erholt haben. Andererseits dürften sich mit fortschreitender Globalisierung ineffiziente akademische Strukturen und Marktabschottung zunehmend negativ auf die Forschungsproduktivität auswirken.

Eine weitergehende Analyse des Einflusses der Ökonomen der verschiedenen Länder wird durch zwei Eigenheiten der heute verfügbaren Daten erschwert. Zum einen wird in den bisherigen Analysen vernachlässigt, dass die Länder unterschiedlich gross sind. Zum anderen beruhen insbesondere die grossen Datensätze von Blaug $(1986,1999)$ auf den individuellen Zitierungen über lange Zeiträume (12 bzw. 14 Jahre), so dass jüngere und heute erfolgreiche Ökonomen schlechtere Chancen haben, erfasst zu werden.

Deshalb misst dieser Aufsatz die Publikationsaktivität und den Einfluss der in in den verschiedenen europäischen Ländern lebenden Ökonomen anhand der Publikationen in einer Auswahl von führenden Fachzeitschriften und den Zitierungen gemäss Social Sciences Citation Index (SSCI) - relativ zur Bevölkerungsgrösse für die jüngeren und kürzeren Zeiträume von 1986-1990 und 1993-1996, die aufgrund der Datenverfügbarkeit ausgewählt wurden. Diese Ergebnisse werden denjenigen von Blaug (1986) gegenübergestellt, die sich auf einen früheren Zeitraum (1972-83) beziehen. Die standardisierten Daten zeigen, dass die internationale Publikationsaktivität und der Einfluss der Ökonomen aus den kleinen Ländern ganz besonders gross sind. Wie unsere Daten erhoben wurden, wird im nächsten Abschnitt beschrieben. Im dritten Abschnitt werden die Ergebnisse vorgestellt, und im vierten Abschnitt mögliche Erklärungen diskutiert. Im letzten Abschnitt werden einige Schlussfolgerungen gezogen.

\section{Daten}

\section{A. Publikationsaktivität}

Die Publikationsaktivität erfassen wir anhand der Anzahl der in ausgewählten Fachzeitschriften publizierenden Autoren eines Landes. Die Beschränkung auf Fachzeitschriften hat den Vorteil, dass sich damit automatisch eine gewisse Standardisierung der berücksichtigten Werke ergibt; beim Vergleich mit Büchern und weiteren Publikationen würde allzu Ungleiches einander gegenübergestellt. Eine Auszählung aller Aufsätze in Fachzeitschriften und aller Zitate 


\section{Ökonomen, Publikationen und Zitationen}

aller europäischen Ökonomen wäre eine Titanenarbeit. Da uns aber nicht die Gesamtzahl aller Publikationen und Zitate interessiert, sondern der relative Anteil der verschiedenen Länder, reicht für unsere Zwecke eine angemessen gewählte Stichprobe. Um die länder- und themenspezifischen Verzerrungen möglichst klein zu halten, konzentrierten wir uns auf international ausgerichtete, inhaltlich breit orientierte Zeitschriften, die einen möglichst grossen Einfluss aufweisen (d.h. deren Aufsätze häufig zitiert werden). So werteten wir schliesslich alle Beiträge aus, die von in Europa tätigen Ökonomen in den Zeiträumen von 1988-1990 und 1993-1996 in den folgenden Zeitschriften publiziert wurden: American Economic Review, Journal of Political Economy, Quarterly Journal of Economics, Econometrica, Review of Economics and Statistics, Review of Economic Studies, European Economic Review, Economic Journal und Kyklos. ${ }^{2}$ Dies sind die nach Zitierungen führenden amerikanischen und europäischen, inhaltlich breiten Zeitschriften (für eine Rangliste des Einflusses der ökonomischen Fachzeitschriften siehe z.B. Figlio 1994, Laband und Piette 1994). So fanden wir für den Zeitraum von 1988-1990 509 und für 1993-1996 813 europäische Ökonomen, die mindestens einen Beitrag in einer dieser neun Zeitschriften veröffentlichen konnten.

\section{B. Einfluss}

Die wissenschaftliche Bedeutung von Einzelpersonen sowie von Zeitschriften und wissenschaftlichen Institutionen wird heute zumeist anhand der Anzahl der Zitierungen zu erfassen versucht. ${ }^{3} \mathrm{Um}$ nicht allzu unterschiedlich gewichtige Zitierungen aufzusummieren, macht es Sinn, nur Zitate in anerkannten wissenschaftlichen Periodika zu berücksichtigen. Unsere Untersuchung stützt sich deshalb Blaug (1986) folgend auf den Social Sciences Citation Index (SSCI). Dieser sammelt die Zitate aus über 220 wirtschaftswissenschaftlichen Fachzeitschriften aus allen Sprachräumen systematisch. ${ }^{4}$ Gezählt werden alle Zitate, gleich ob in Fachzeitschriften erschienene Artikel, Kommentare und Entgegnungen oder

2. Die Review of Economic Studies wurde nur für den Zeitraum von 1993-1996 berücksichtigt.

3. Für ökonomische Fachzeitschriften siehe z.B. Liebowitz und Palmer (1984), Laband und Piette (1994), Stigler, Stigler und Friedland (1995), Burton und Phimister (1995), Hodgson und Rothman (1999); für wissenschaftliche Institutionen siehe z.B. Laband (1985), Conroy et al. (1995), Dusansky und Vernon (1998), Hodgson und Rothman (1999); für vertiefte Analysen einzelner Länder siehe z.B. Pommerehne und Frey (1988) für Deutschland oder Meier (1994) für die Schweiz; für Untersuchungen einzelner ökonomischer Subdisziplinen siehe z.B. Steinmann, Olbrich und Riedl (1998). Dieses Verfahren ist auch in anderen Wissenschaften üblich, vgl. z.B. für die Psychologie Keul, Gigerenzer und Stroebe (1993) (ebenfalls aufgrund des Social Sciences Citation Index), oder für verschiedenste Naturwissenschaften (aufgrund des Science Citation Index) Strate, Winterhager und Sehringer (1991) für eine vertiefte Analyse des Outputs in einem Land und Woolgar (1991) für eine Übersicht und weitere Literaturhinweise.

4. Neben den über 220 ökonomischen Fachzeitschriften werden rund 1350 sozialwissenschaftliche Zeitschriften vollständig und weitere rund 2150 nicht-schwergewichtig sozialwissenschaftliche Periodika selektiv ausgewertet, d.h. nur die Zitierungen mit sozialwissenschaftlichem Inhalt erfasst. 


\section{Reiner Eichenberger, Ursina Meier und Rolf Arpagaus}

Aufsätze in Sammelbänden oder Monographien zitiert werden. Dabei wird nicht zwischen Beiträgen eines Alleinautors und Gemeinschaftswerken unterschieden. Für gewisse Fragen nachteilig ist, dass nur die Erstautoren von zitierten Gemeinschaftswerken angeführt und nur die Initialen der Vornamen der zitierten Autoren angegeben werden, so dass es gelegentlich aufwendig ist, die Zitate den verschiedenen Autoren gleichen (gekürzten) Namens zuzuordnen. Diese Eigenheiten erschweren zwar Analysen der Zitierungen einzelner Autoren; für vergleichende Aussagen auf der Ebene von Ländern dürften sie jedoch kaum eine Rolle spielen. ${ }^{5}$

Den Einfluss der Ökonomen aus den verschiedenen Ländern für 1986-1990 messen wir, indem wir zuerst die Zitierungen aller 509 Ökonomen aus unserer Publikationsaktivitätsliste für 1988-1990 und aller in Blaug angeführten 209 im Jahre 1985 in Europa lebenden Autoren anhand eines Fünfjahres-Band des Social Sciences Citation Index (SSCI 1992) auszählen, der die Zitierungen aus den Jahren 1986-1990 erfasst. Den Einfluss für 1993-1996 ermitteln wir, indem wir in den entsprechenden SSCI-Jahresbänden die Zitierungen aller Ökonomen auszählen, die auf unserer Publikationsaktivitätsliste für diesen Zeitraum, oder auf der Publikationsaktivitätsliste für 1988-1990, oder in Blaug (1986) angeführt werden. Dieser Selektionsmechanismus ist geeignet, die Meistzitierten zu erfassen: Während durch Blaug die zwischen 1972 und 1982 bereits vielzitierten, d.h. früher besonders aktiven Autoren erfasst werden, zielen die anderen Listen auf die in der zweiten Hälfte der 80er und in den 90er Jahren besonders aktiven Ökonomen. Um den Einfluss der Ökonomen der verschiedenen Länder festzustellen, selektionieren wir für die zwei Zeiträume von 1986-1990 und 19931996 die im Januar 1990 bzw. die im Januar 1995 in Europa lebenden Ökonomen, die in der betreffenden Periode über hundertmal zitiert werden. Da nicht auszuschliessen ist, dass unser Selektionsmechanismus einzelne wichtige Autoren nicht erfasst, haben wir für den Zeitraum von 1986-1990 die Zitierungen einer grossen Zahl weiterer besonders aktiver Ökonomen und Ökonominnen, insbesondere vieler Mitglieder der European Economic Association und des Vereins für Socialpolitik, nachgezählt. Dabei erwies sich, dass durch unseren Selektionsmechanismus nur ganz wenige Autoren nicht erfasst werden, die ebenfalls zu den Meistzitierten (d.h. mit über 100 Zitaten) zählen. ${ }^{6}$ So ergeben sich schliesslich die zwei Listen der Meistzitierten mit für 1986-1990 126 und für 1993-1996 161 Ökonomen. Es ist hier aber nochmals zu betonen, dass mit unserer Methode keine vollständige Erfassung der Publikationen und Zitierungen aller europäischen Ökonomen gelungen sein kann; dies war auch nicht Ziel der Erhebung. Für unseren Zweck - eine vergleichende Analyse auf Ebene der europäischen Länder dürften die Daten aber sehr wohl eine verlässliche Grundlage bilden.

5. Unklarheiten gab es fast ausschliesslich für britische Autoren. Da wir in den wenigen nicht mit Sicherheit klärbaren Zweifelsfällen die Zitierungen nicht gezählt haben, wirkt diese (minime) Verzerrung der britischen Dominanz entgegen.

6. Für den Zeitraum von 1993-1996 haben wir auf diese subjektive Kontrollprozedur verzichtet, da sie für den früheren Zeitraum kaum relevante Veränderungen bewirkt hat. 


\section{Ökonomen, Publikationen und Zitationen}

\section{Ergebnisse}

\section{A. Nach Ländern}

Nicht standardisierte Beobachtungen

Eine Übersicht über die Ergebnisse gibt Tabelle 1. Sie zeigt den jeweiligen prozentualen Anteil der Autoren der einzelnen Länder an den verschiedenen Gesamtheiten: Die zweite und dritte Spalte beziehen sich auf die von uns erfassten 813 und 509 Autoren, die von 1993-1996 und 1988-1990 in einer der neun bzw. acht ausgezählten führenden Zeitschriften wenigstens eine Veröffentlichung plazieren konnten. Die vierte und fünfte Spalte geben Auskunft über die mit dem beschriebenen Selektionsprozess gefundenen 161 und 126 Ökonomen, die 1993-1996 und 1986-1990 mehr als hundertmal zitiert werden. Die sechste Spalte bezieht sich auf die 209 von Blaug aufgeführten, von 1972-1983 am häufigsten zitierten im Jahre 1985 in Europa lebenden Wissenschaftler. In der siebten Spalte schliesslich werden die von Blaug angeführten, vor 1985 verstorbenen europäischen Ökonomen den Ländern zugeordnet.

In allen Datensätzen ist die Dominanz von Ökonomen aus Grossbritannien augenfällig. Insofern sind sie den Ergebnissen von Blaug (1986) und Pommerehne und Frey (1988), aber auch neueren Auswertungen wie Kalaitzidakis, Mamuneas und Stengos (1999) ähnlich. Verglichen mit Blaugs (1986) Daten für lebende Ökonomen schneiden Frankreich, Italien und auch Deutschland relativ zu Grossbritannien schlechter, ${ }^{7}$ die anderen europäischen Länder hingegen leicht besser ab.

\section{Nach Bevölkerungszahl standardisierte Daten}

Die bisher vorgestellten Zahlen sind mit einem Mangel behaftet, der jeden sinnvollen Ländervergleich erschwert: Die Länder sind von ganz unterschiedlicher Grösse. Deshalb werden in Tabelle 2 bezüglich der Bevölkerungsgrösse standardisierte Daten wiedergegeben. Dazu werden die Daten aus Tabelle 1 durch die Bevölkerungszahl in Millionen geteilt (für Blaugs Daten wurde die Bevölkerungsgrösse von 1980, für unsere eigenen Daten diejenige von 1990 und 1995 verwendet). Dadurch ergibt sich ein zwischen Ländern und zwischen den verschiedenen Datensätzen vergleichbarer Index, der Antwort darauf gibt, wieviele Prozentpunkte des Anteils an allen publizierenden und häufig zitierten Autoren pro Million Einwohner des jeweiligen Landes erbracht werden.

Die standardisierten Publikationsaktivitäts- und Einflussmasse ergeben ein völlig neues Bild, das sich stark von den Resultaten von Pommerehne und Frey

7. Die gleiche Entwicklung zeigt sich bei einem Vergleich der zweiten und dritten Auflage des Who's Who in Economics, die auf Zitierungen von 1972-1983 bzw. 1984-1996 beruhen. Die Anteile an der Gesamtheit der vielzitierten europäischen Ökonomen sinken für Frankreich von 12,4 auf 9,5, für Deutschland von 9,6 auf 8,0, und für Italien von 4,8 auf 4,6 Prozent, während Grossbritanniens Anteil von 58,4 auf 63,5 Prozent zunimmt. 


\begin{tabular}{|c|c|c|c|c|c|c|}
\hline Länder & $\begin{array}{l}\text { Publikations- } \\
\text { aktivität } \\
\text { 1993-1996 } \\
(\mathrm{n}=813)\end{array}$ & $\begin{array}{l}\text { Publikations- } \\
\text { aktivität } \\
\text { 1988-1990 } \\
(\mathrm{n}=509)\end{array}$ & $\begin{array}{l}\text { Einfluss } \\
\text { 1993-1996 } \\
(\mathrm{n}=161)\end{array}$ & $\begin{array}{c}\text { Einfluss } \\
1986-1990 \\
(\mathrm{n}=126)\end{array}$ & $\begin{array}{c}\text { Einfluss } \\
1972-1983 \\
\text { nach Blaug }^{\mathrm{a}} \\
(\mathrm{n}=209)\end{array}$ & $\begin{array}{c}\text { Einfluss } \\
1985 \\
\text { verstorb. Ökonomen } \\
\text { nach Blaug }{ }^{\mathrm{a}}(\mathrm{n}=332)\end{array}$ \\
\hline Grossbritannien & 40,1 & 42,9 & 56,5 & 66,6 & 58,4 & 46,7 \\
\hline Frankreich & 9,7 & 9,2 & 8,1 & 7,1 & 12,4 & 14,5 \\
\hline Niederlande & 8,9 & 7,3 & 3,7 & 3,2 & 4,3 & 0,6 \\
\hline Deutschland & 8,0 & 10,6 & 5,6 & 4,0 & 9,6 & 16,0 \\
\hline Belgien & 5,5 & 6,1 & 6,2 & 2,4 & 2,4 & 0,9 \\
\hline Spanien & 5,2 & 2,2 & 1,9 & 0 & 0,5 & 0,9 \\
\hline Italien & 4,9 & 4,7 & 5,0 & 4,0 & 4,8 & 6,0 \\
\hline Schweiz & 4,3 & 3,9 & 4,4 & 4,8 & 1,9 & 1,5 \\
\hline Schweden & 3,7 & 3,1 & 3,1 & 2,4 & 1,4 & 2,4 \\
\hline Österreich & 2,6 & 2,2 & 0,6 & 0 & 0,5 & 3,3 \\
\hline Norwegen & 2,0 & 1,8 & 1,2 & 2,4 & 1,9 & 0,6 \\
\hline Finnland & 1,0 & 0,4 & 0 & 0 & 0 & 0 \\
\hline Dänemark & 0,9 & 1,6 & 1,2 & 0 & 0 & 0,3 \\
\hline Irland & 0,9 & 0,4 & 0,6 & 0,8 & 0,5 & 2,4 \\
\hline Portugal & 0,7 & 0,8 & 0 & 0 & 0 & 0 \\
\hline Ungarn & 0,4 & 1,0 & 0,6 & 1,6 & 0,5 & 0,3 \\
\hline Griechenland & 0,4 & 0,2 & 0,6 & 0 & 0 & 0 \\
\hline Slovenien $^{\mathrm{b}}$ & 0,2 & 0,6 & 0 & 0,8 & 0 & 0 \\
\hline Russland $^{\mathrm{C}}$ & 0,2 & 0,2 & 0 & 0 & 1,0 & 3,0 \\
\hline Luxemburg & 0,2 & 0,2 & 0 & 0 & 0 & 0 \\
\hline Island & 0,1 & 0,2 & 0,6 & 0 & 0 & 0 \\
\hline Tschechien $^{\mathrm{d}}$ & 0,1 & 0,6 & 0 & 0 & 0 & 0 \\
\hline Polen & 0 & 0,2 & 0 & 0 & 0 & 0,6 \\
\hline
\end{tabular}

${ }^{a}$ Eigene Berechnungen anhand von Blaug (1986); ${ }^{b}$ Werte vor 1990 für Jugoslawien; ${ }^{c}$ Werte vor 1990 für Sowjetunion; ${ }^{\mathrm{d}}$ Werte vor 1990 für Tschechoslowakei.

Quelle: Eigene Auswertungen verschiedener Fachzeitschriften sowie Berechnungen aufgrund des Social Sciences Citation Index und Frey und Pommerehne (1988). 


\section{Ökonomen, Publikationen und Zitationen}

(1988) aufgrund der Daten von Blaug (1986) sowie neuerer Publikationen wie Kalaitzidakis, Mamuneas und Stengos (1999) unterscheidet.

\section{Standardisierte Publikationsaktivität}

Bei der Publikationsaktivität sind fünf Ländergruppen auszumachen: (i) Grossbritannien, das für alle sechs Masszahlen die höchsten Werte erreicht. (ii) Die kleinen westeuropäischen Länder Belgien, Dänemark, Finnland, Irland, Island, Luxemburg, Niederlande, Norwegen, Österreich, Schweden und die Schweiz: Sie erreichen im (mit der Bevölkerungsgrösse gewichteten) Durchschnitt einen standardisierten Publikationswert von 0,44 für 1993-1996 und von 0,41 für 1988-1990 und damit 64 Prozent bzw. 55 Prozent des britischen Wertes. Unter Berücksichtigung des jeweils höheren Wertes aus den zwei Perioden weisen sie alle ausnahmslos zwischen 28 Prozent und 90 Prozent des britischen Publikationsaktivitätsniveaus auf. (iii) Die drei grossen Länder Deutschland, Frankreich und Italien: Sie erreichen im gewichteten Durchschnitt einen Publikationswert von 0,12 für 1993-1996 und 0,14 für 1988-1990, also rund 17 Prozent und 19 Prozent des britischen Wertes. Keines dieser Länder erzielt je mehr als 25 Prozent des britischen Publikationsaktivitätsniveaus. (iv) Die südeuropäischen Länder Griechenland, Portugal und Spanien: Sie erreichen im gewichteten Durchschnitt 0,10 für 1993-1996 und 0,06 für 1988-1990 oder rund 14 Prozent und 8 Prozent des britischen Publikationsaktivitätsniveaus. Keines von ihnen erzielt je mehr als 19 Prozent des britischen Wertes. (v) Die osteuropäischen Staaten schliesslich sind noch weiter vom Bereich des britischen Publikationsaktivitätsniveaus entfernt. $\mathrm{Zu}$ berücksichtigen bleibt, dass die Werte einiger kleiner Staaten von wenigen Beobachtungen abhängen.

Der bisher in der Literatur kaum bestrittene Vorsprung Grossbritanniens in der Publikationsaktivität erscheint viel kleiner, sobald bezüglich der Bevölkerungsgrösse standardisierte Masszahlen zum Vergleich herangezogen werden. Zudem ist dieser Vorsprung in der von uns erhobenen Publikationsaktivität wesentlich geringer als in Blaugs Ergebnissen für den Einfluss, die auf wesentlich kleineren Zahlen von Ökonomen beruhen. So erreichen Belgien (mit 45 von insgesamt 813 und 31 von 509 der von uns für die beiden Zeitperioden ermittelten Ökonomen), die Schweiz (mit 35 und 20) und die Niederlande (mit 72 und 37) alle immer wenigstens 65 Prozent der standardisierten Publikationsaktivität Grossbritanniens, was bei den jeweiligen Nennungen pro Land sicher kein Kleinzahlenphänomen darstellt. Bemerkenswert ist auch die Entwicklung der Publikationsaktivität über die zwei Zeiträume. In acht der elf kleinen westeuropäischen Ländern nimmt sie sowohl relativ zu allen anderen Ländern wie auch relativ zu Grossbritannien zu. Im Zeitraum von 1993-1996 schliessen Belgien mit 80 Prozent, die Niederlande mit 83 Prozent und die Schweiz mit sogar 90 Prozent fast zu den Briten auf. Aber auch die grossen Länder und die südeuropäischen Länder haben (mit Ausnahme Deutschlands) zwischen 19881990 und 1993-1996 gegenüber Grossbritannien leicht aufgeholt. Verglichen mit den Daten für den von Blaug erfassten früheren Zeitraum haben jedoch nur die kleinen westeuropäischen und die südeuropäischen Länder aufgeholt. Die 
Tabelle 2 Publikationsaktivität und Einfluss nach Ländern, nach Bevölkerungsgrösse standardisierte Anteile

\begin{tabular}{|c|c|c|c|c|c|c|}
\hline Länder & $\begin{array}{c}\text { standard. } \\
\text { Publikations- } \\
\text { aktivität } \\
\text { 1993-1996 } \\
(\mathrm{n}=813)\end{array}$ & $\begin{array}{c}\text { standard. } \\
\text { Publikations- } \\
\text { aktivität } \\
1988-1990 \\
(\mathrm{n}=509)\end{array}$ & $\begin{array}{l}\text { standard. } \\
\text { Einfluss } \\
\text { 1993-1996 } \\
(\mathrm{n}=161)\end{array}$ & $\begin{array}{l}\text { standard. } \\
\text { Einfluss } \\
\text { 1986-1990 } \\
(\mathrm{n}=126)\end{array}$ & $\begin{array}{c}\text { standard. } \\
\text { Einfluss } \\
1972-1983 \\
\text { nach Blaug }^{\mathrm{a}} \\
(\mathrm{n}=209)\end{array}$ & $\begin{array}{c}\text { standard. } \\
\text { Einfluss } \\
1985 \text { verstorb. }^{\text {Ookomen }}{ }^{\mathrm{a}} \\
\text { Ökonom }^{(\mathrm{n}=332)}\end{array}$ \\
\hline Grossbritannien & 0,69 & 0,75 & 0,97 & 1,16 & 1,04 & 0,83 \\
\hline Belgien & 0,55 & 0,61 & 0,61 & 0,24 & 0,24 & 0,09 \\
\hline Dänemark & 0,17 & 0,31 & 0,24 & 0 & 0 & 0,06 \\
\hline Finnland & 0,19 & 0,08 & 0 & 0 & 0 & 0 \\
\hline Irland & 0,24 & 0,11 & 0,17 & 0,23 & 0,15 & 0,71 \\
\hline Island & 0,46 & 0,67 & 2,3 & 0 & 0 & 0 \\
\hline Luxemburg & 0,60 & 0,50 & 0 & 0 & 0 & 0 \\
\hline Niederlande & 0,57 & 0,49 & 0,24 & 0,22 & 0,31 & 0,04 \\
\hline Norwegen & 0,45 & 0,43 & 0,29 & 0,57 & 0,48 & 0,15 \\
\hline Österreich & 0,32 & 0,29 & 0,08 & 0 & 0,07 & 0,44 \\
\hline Schweden & 0,42 & 0,36 & 0,35 & 0,28 & 0,17 & 0,29 \\
\hline Schweiz & 0,62 & 0,58 & 0,62 & 0,72 & 0,30 & 0,24 \\
\hline Deutschland $^{\mathrm{b}}$ & 0,10 & 0,17 & 0,07 & 0,06 & 0,16 & 0,26 \\
\hline Frankreich & 0,17 & 0,16 & 0,14 & 0,13 & 0,23 & 0,27 \\
\hline Italien & 0,09 & 0,08 & 0,09 & 0,07 & 0,09 & 0,11 \\
\hline Griechenland & 0,04 & 0,02 & 0,06 & 0 & 0 & 0 \\
\hline Portugal & 0,07 & 0,08 & 0 & 0 & 0 & 0 \\
\hline Spanien & 0,13 & 0,06 & 0,05 & 0 & 0,01 & 0,02 \\
\hline Slovenien $^{\mathrm{c}}$ & 0,12 & 0,03 & 0 & 0,03 & 0 & 0 \\
\hline Polen & 0,00 & 0,01 & 0 & 0 & 0 & 0,02 \\
\hline Russland $^{\mathrm{d}}$ & 0,002 & 0,0007 & 0 & 0 & 0,01 & 0,01 \\
\hline Tschechien ${ }^{\mathrm{e}}$ & 0,01 & 0,04 & 0 & 0 & 0 & 0 \\
\hline Ungarn & 0,04 & 0,10 & 0,06 & 0,15 & 0,05 & 0,03 \\
\hline
\end{tabular}

${ }^{a}$ Eigene Berechnungen anhand von Blaug (1986); ${ }^{\mathrm{b}}$ für 1988-1990 auf alte Bundesländer standardisiert; die Werte von 1993-1996 auf die alten Bundesländer standardisiert sind für die Publikationsaktivität 0,13 und für den Einfluss 0,09; ${ }^{\mathrm{c}}$ Werte vor 1990 für Jugoslawien; ${ }^{\mathrm{d}}$ Werte vor 1990

$\tilde{\Xi} \quad$ für Sowjetunion; ${ }^{\mathrm{e}}$ Werte vor 1990 für Tschechoslowakei. Pommerehne (1988). 


\section{Ökonomen, Publikationen und Zitationen}

grossen Länder Deutschland, Frankreich und Italien hingegen fielen tendenziell weiter zurück oder stagnierten.

\section{Standardisierter Einfluss}

Beim Einfluss zeigt sich ein ganz ähnliches Bild, wenn auch dieses Mass infolge der kleineren Gesamtzahl von erfassten Autoren ( $n=161$ für 1993-1996 und $\mathrm{n}=126$ für 1986-1990) stärker von Kleinzahlenphänomenen geprägt ist als diejenigen für die Publikationsaktivität $(\mathrm{n}=813 \mathrm{bzw}$. 509) oder die Masse von Blaug ( $\mathrm{n}=209$ bzw. 332). Insgesamt zeigt sich aber auch in unseren Einflussdaten (Zitierungen) ein klarer Vorsprung Grossbritanniens vor allen anderen europäischen Ländern, der noch deutlicher ausfällt als bei der Publikationsaktivität. Eine Erklärung dafür wäre, dass die britischen Ökonomen nicht nur mehr publizieren, sondern ihre Beiträge dann auch noch häufiger zitiert werden als diejenige anderer europäischen Ökonomen. Bemerkenswerterweise kommt jedoch der standardisierte Einfluss der Ökonomen einiger kleiner Länder den Briten recht nahe. Dies gilt insbesondere für Belgien mit 63 Prozent des britischen Einflusses für 1993-1996 (aber nur 21 Prozent für 1986-1990) und die Schweiz mit 64 Prozent für 1993-1996 und 62 Prozent für 1986-1990. Auffällig ist wiederum, dass der britische Einfluss in ähnlichem Ausmass wie die Publikationsaktivität von 1986-1990 bis 1993-1996 abgenommen hat. Entsprechend hat der Einfluss der anderen Ländergruppen (ausser Osteuropa) leicht zugenommen.

Was die Publikationsaktivität und den Einfluss von in Süd- und in Osteuropa lebenden Ökonomen betrifft, offenbaren unsere Ergebnisse keine grossen Überraschungen. Augenfällig ist zwar, dass Portugal und Spanien bei den Publikationen fast mit Italien gleichzuziehen vermögen und vor allem der Beitrag ungarischer Ökonomen aus demjenigen ihrer ost- und südeuropäischen Kollegen herausragt. Da diese Daten aber zumeist auf sehr wenigen Beobachtungen beruhen, werden sie hier nicht weiter interpretiert.

Der Unterschied zwischen den kleinen westeuropäischen Ländern und den drei grossen Ländern Deutschland, Frankreich und Italien verdient besondere Beachtung. Die elf kleinen Länder weisen wie bei der Publikationsaktivität auch beim Einfluss (Zitierungen) einen grossen Vorsprung auf. Dies trifft neben Norwegen, Schweden und der Schweiz auch für das etwas grössere Belgien und die Niederlande zu. Während die einzelnen Werte für die anderen kleinen Länder infolge der kleinen Beobachtungszahlen keine grosse Aussagekraft besitzen, zeigen die Zahlen für die beiden Ländergruppen eindrückliche Unterschiede. Im Zeitraum 1986-1990 brachten die elf kleinen Länder mit einer Gesamtbevölkerung von 66,4 Mio. insgesamt 132 (25,9 Prozent) der 509 Autoren der von uns erfassten Publikationen und 20 (15,9 Prozent) der 126 meistzitierten Ökonomen auf; hingegen stellen die drei grossen Länder Deutschland, Frankreich und Italien mit einer fast dreimal so grossen Gesamtbevölkerung von 177,7 Mio. nur 125 Autoren (24,6 Prozent) und 19 (15,1 Prozent) der meistzitierten Ökonomen. Im Zeitverlauf entwickelten sich die Verhältnisse sogar noch zulasten der grossen Länder. Im Zeitraum 1993-1996 


\section{Reiner Eichenberger, Ursina Meier und Rolf Arpagaus}

stellten die elf kleinen Länder mit einer Gesamtbevölkerung von dann 68,3 Mio. zusammen schon 244 (30,0 Prozent) der insgesamt 813 Autoren und 35 (21,7 Prozent) der 161 meistzitierten Ökonomen; die grossen Länder zusammen mit ihrer dann 204,3 Mio. zählenden Bevölkerung brachten es jedoch nur auf 184 (22,6 Prozent) Autoren und 30 (18,6 Prozent) der meistzitierten Ökonomen.

Für den Einfluss zeigt sich unter Einbezug von Blaugs Auswertung für die kleinen westeuropäischen Ländern eine stetige Zunahme von 0,19 (vor 1985 verstorbene Ökonomen) über 0,21 (1972-1983) und 0,24 (1986-1990) auf 0,32 (1993-1996); im Gegensatz dazu nahm der Einfluss der grossen Länder von 0,21 (vor 1985 verstorbene Ökonomen) über 0,16 (1972-1983) auf 0,09 (19861990) ab, und dann nur ganz leicht wieder auf 0,10 (1993-1996) zu.

Wie die bisherigen Ergebnisse zeigen, hat die scheinbare Dominanz britischer Ökonomen zwei Ursachen. Der überaus deutliche Abstand gegenüber den grossen kontinentaleuropäischen Ländern kommt dadurch zustande, dass die Briten viel häufiger in internationalen Fachzeitschriften publizieren und ihre Beiträge dann wohl auch noch öfter zitiert werden. Der viel geringere Vorsprung gegenüber den kleinen europäischen Ländern hingegen beruht zu einem grossen Teil darauf, dass Grossbritannien einfach grösser ist als diese Länder. Die auf die Landesgrösse standardisierten Daten zeigen, dass die Ökonomen aus kleinen Ländern hinsichtlich ihrer internationalen Publikationsaktivität den Briten nur wenig nachstehen.

\section{B. Personen}

Manchen Leser mag natürlich auch eine personenspezifische Auswertung unserer Zitierungs- bzw. Einflussdaten interessieren. Wie bereits erwähnt, konnte unsere Erfassung der verschiedenen Autoren nicht vollständig sein. Die Zitierungen von Wissenschaftlern, die in den Zeiträumen von 1988-1990 und 1993-1996 in keiner der führenden allgemeinen Fachzeitschriften publiziert haben, die auch nicht in Blaugs Who's Who in Economics Erwähnung finden und die überdies unserer Nachauswertung besonders bekannter und aktiver Mitglieder der European Economic Association und anderer uns besonders gewichtig erscheinenden Ökonomen entgangen sind, wurden nicht ausgezählt. Unserem Selektionsverfahren ist jedoch eigen, dass die besonders bekannten und aktiven Autoren auch besonders vollständig erfasst werden. Weil überdies die Auswertung für den Zeitraum von 1993-1996 umfassender ist, gehen wir im folgenden nur auf die 20 meistzitierten Autoren dieser Periode ein.

Diese Autoren, allesamt männlichen Geschlechts, wurden im betreffenden Zeitraum von 1993-1996 zwischen 367 und 1232 mal zitiert. Neun dieser zwanzig Ökonomen werden sowohl bei Blaug (1986) angeführt wie auch durch unser Publikationsselektionsverfahren erfasst; ${ }^{8}$ drei Wissenschaftler werden nur

8. S.J. Nickell wird von Blaug (1986) nicht im Hauptteil, sondern nur in Appendix 4 (unter denjenigen Autoren, mit denen Blaug keinen Kontakt aufnehmen konnte) erwähnt. 
Ökonomen, Publikationen und Zitationen

Tabelle 3 Die zwanzig meistzitierten europäischen Ökonomen, 1993-1996

\begin{tabular}{lccc}
\hline Name & Jahrgang & Land & Zitierungen \\
\hline Søren Johansen & 1939 & DK & 1232 \\
Anthony B. Atkinson & 1944 & GB & 898 \\
Partha S. Dasgupta & 1942 & GB & 775 \\
Janos Kornai & 1928 & H & 753 \\
David F. Hendry & 1944 & GB & 708 \\
Jean Tirole & 1953 & F & 657 \\
Jean-Jacques Laffont & 1947 & F & 567 \\
Ken Binmore & 1940 & GB & 544 \\
Andrew Harvey & 1947 & GB & 523 \\
John H. Dunning & 1927 & Ö & 518 \\
Dennis C. Mueller & 1940 & I & 515 \\
Giovanni Dosi & 1953 & D & 502 \\
Reinhard Selten & 1930 & GB & 494 \\
David W. Pearce & 1941 & CH & 457 \\
Bruno S. Frey & 1941 & GB & 405 \\
Stephen J. Nickell & 1944 & GB & 397 \\
Philippe Aghion & 1956 & GB & 392 \\
Mark Blaug & 1927 & GB & 388 \\
M. Hashem Pesaran & 1948 & NL & 370 \\
Angus Maddison & 1926 & 367 \\
\hline
\end{tabular}

Quelle: Eigene Auswertung des Social Sciences Citation Index.

durch Blaug angeführt; und sieben werden ausschliesslich durch unsere Publikationslisten genannt. Nur ein Autor wird weder von Blaug noch in unseren Publikationsdaten angeführt, sondern wurde erst im Laufe unserer ausgedehnten Nachzählungen ermittelt. Dies ist mit Reinhard Selten als Nobelpreisträger zwar ein besonders prominentes Mitglied dieser Gruppe; allerdings ist Selten für sein zurückhaltendes Publikationsverhalten bekannt, was wohl auch der Grund dafür ist, dass er sogar bei Blaug (1986) keine Erwähnung findet. Die Ergebnisse unserer Nachzählungen lassen hoffen, dass er der einzige wirklich einflussreiche Ökonom ist, der unserem Selektionsmechanismus (ohne Nachzählungen) entgangen ist.

Auch unter diesen zwanzig meistzitierten Ökonomen zeigt sich ein deutliches britisches Übergewicht. Elf von ihnen waren an britischen Universitäten oder Institutionen verpflichtet, zwei waren in Frankreich, und je einer in Deutschland, Dänemark, Italien, Österreich, der Schweiz und Ungarn tätig. Relativ zur Bevölkerung zeigt sich hier ein ähnliches Muster wie in den grösseren Datenfeldern, die oben vorgestellt wurden. Die grossen Länder bringen trotz ihrer gut dreimal grösseren Bevölkerungszahl gleich viel eminente Ökonomen wie die kleinen westeuropäischen Länder auf, nämlich vier. Die geographische Konzentration kontrastiert mit der fachlichen Vielfalt, für die diese zwanzig Ökonomen stehen. So sind Anthony Atkinson mit 


\section{Reiner Eichenberger, Ursina Meier und Rolf Arpagaus}

Verteilungstheorie und Sozialpolitik, Partha Dasgupta mit u.a. Entwicklungspolitik und Ressourcenökonomie, Janos Kornai mit der ökonomischen Analyse sozialistischer Systeme, Jean Tirole und Jean-Jacques Laffont mit Industrial Organization, Ken Binmore mit Spieltheorie, John Dunning mit Internationalen Wirtschaftsbeziehungen, Dennis Mueller und Bruno Frey mit politischer (und aussermarktlicher) Ökonomie, Giovanni Dosi mit evolutionärer Ökonomie, Reinhard Selten mit experimenteller Ökonomie, D.W. Pearce mit Umweltökonomie, Stephen Nickell mit Arbeitsmarkttheorie, Philippe Aghion mit Wachstumstheorie (und Industrial Organization), Mark Blaug mit Dogmengeschichte und Angus Maddison mit Wirtschaftsgeschichte sowie die Ökonometriker Søren Johansen (Kointegration), David Hendry (Philosophy of the London School), Andrew Harvey (Kalman Filter) und Hashem Pesaran (Zeitreihen- und Panelanalyse) international führende Vertreter ganz unterschiedlicher Spezialisierungen.

\section{Robustheit der Ergebnisse}

Da unsere Ergebnisse auf der Auswertung von neun Fachzeitschriften aufbauen, stellt sich natürlich die Frage, ob unsere Resultate ungebührlich durch die Auswahl dieser Zeitschriften beeinflusst wurden. So erscheint es denkbar, dass die Autorenzusammensetzung des Economic Journal (der Zeitschrift der Royal Economic Association) oder von Kyklos (einer in der Schweiz herausgegebenen sozialwissenschaftlichen Zeitschrift) die Ergebnisse stark beeinflussen.

Um abzuklären, inwiefern unsere Ergebnisse von der Auswahl der Zeitschriften abhängen, haben wir die Publikationsaktivität für 1993-1996 jeweils unter Weglassung einer der neun Zeitschriften ermittelt. In Tabelle 4 werden die Ergebnisse für Grossbritannien, die kleinen westeuropäischen Länder und die drei grossen Länder Deutschland, Frankreich und Italien dargestellt.

Offensichtlich beeinflusst die Auswahl der Zeitschriften die erfasste Publikationsaktivität. Dies trifft insbesondere auf Länder zu, deren Ökonomen in absoluten Zahlen gemessen wenige Aufsätze veröffentlichen, vor allem also auf Island und Luxemburg. Das bisher beobachtete Muster bleibt hingegen vollständig erhalten. Grossbritannien weist besonders hohe Minimal- und Maximalwerte auf, und die kleinen westeuropäischen Länder liegen (von Island und Luxemburg einmal abgesehen) sowohl bei den Minimal- als auch bei den Maximalwerten wenigstens gleichauf mit den grossen Ländern, zumeist jedoch weit voraus. Wiederum erreichen Belgien, die Niederlande, Norwegen, Schweden und die Schweiz annähernd die britischen Werte oder übertreffen diese sogar. Dies trifft insbesondere dann zu, wenn Publikationen im Economic Journal nicht berücksichtigt werden. Dann übertreffen Belgien, Island, Luxemburg, die Niederlande, Norwegen und die Schweiz Grossbritannien, und Schweden zieht mit letzterem gleich. ${ }^{9}$

9. Anzumerken bleibt auch, dass die Berücksichtigung der Publikationen in Kyklos das Gesamtergebnis weniger stark beeinflusst als die Publikationen in der European Economic 


\section{Ökonomen, Publikationen und Zitationen}

Tabelle 4 Publikationsaktivität, 1993-1996, nach Bevölkerungsgrösse standardisiert, jeweils unter Weglassung einer Zeitschrift ermittelt

\begin{tabular}{|c|c|c|c|}
\hline Länder & „Normalwert" & $\begin{array}{l}\text { Minimum } \\
\text { (weggelassene } \\
\text { Zeitschrift) }\end{array}$ & $\begin{array}{l}\text { Maximum } \\
\text { (weggelassene } \\
\text { Zeitschrift) }\end{array}$ \\
\hline Grossbritannien & 0,69 & $0,46(\mathrm{EJ})$ & 0,74 (KYK) \\
\hline Belgien & 0,55 & 0,39 (EER) & $0,72(\mathrm{EJ})$ \\
\hline Dänemark & 0,17 & 0,13 (EER) & 0,19 (EJ) \\
\hline Finnland & 0,19 & 0,10 (EER) & $0,24(\mathrm{EJ})$ \\
\hline Irland & 0,24 & 0,15 (AER) & 0,24 (EER) \\
\hline Island & 0,46 & $0,0 \quad(\mathrm{KYK})$ & 0,57 (EER) \\
\hline Luxemburg & 0,60 & $0,0 \quad$ (EER) & $0,75(\mathrm{EJ})$ \\
\hline Niederlande & 0,57 & $0,47(\mathrm{KYK})$ & $0,66(\mathrm{EJ})$ \\
\hline Norwegen & 0,45 & $0,37(\mathrm{ECON})$ & $0,54(\mathrm{EJ})$ \\
\hline Österreich & 0,32 & 0,26 (EER) & 0,39 (EJ) \\
\hline Schweden & 0,42 & 0,36 (EER) & $0,48(\mathrm{KYK})$ \\
\hline Schweiz & 0,62 & 0,52 (KYK) & 0,77 (EJ) \\
\hline Deutschland $^{\mathrm{a}}$ & 0,10 & 0,08 (KYK) & $0,12(\mathrm{EJ})$ \\
\hline Frankreich & 0,17 & 0,15 (EER) & $0,21(\mathrm{EJ})$ \\
\hline Italien & 0,09 & 0,06 (EER) & $0,10(\mathrm{EJ})$ \\
\hline
\end{tabular}

${ }^{a}$ Die Publikationsaktivität auf die alten Bundesländer standardisiert sind 0,13 („,normal“), 0,10 (Minimum), 0,15 (Maximum).

Quelle: Eigene Erhebungen.

\section{$\overline{\text { IV. Erklärungen }}$}

Die vergleichsweise intensive Publikationsaktivität und der starke Einfluss der britischen Ökonomen kann auf verschiedene Weise zu erklären versucht werden.

So kann argumentiert werden, die Daten seien verzerrt, u.a. weil im SSCIIndex schwergewichtig angelsächsische Zeitschriften ausgewertet werden. Diese Erklärung vermag nicht zu befriedigen. Zwar werden im SSCI-Index mehr englischsprachige wirtschaftswissenschaftliche Zeitschriften als nicht-englischsprachige ausgewertet. Trotzdem werden die meisten wichtigen im nichtenglischsprachigen Raum oder nicht in englischer Sprache erscheinenden Zeitschriften berücksichtigt. Es gibt eben mehr international renommierte englischsprachige als nicht-englischsprachige ökonomische Fachzeitschriften, was natürlich wiederum mit den hier diskutierten Aspekten zusammenhängt.

Eine andere, oft angeführte Erklärung thematisiert die sprachlichen Eintrittsbarrieren, denen sich nicht-englischsprachige Autoren gegenüber sehen, wenn sie in den internationalen Wissenschaftsmarkt eintreten wollen

Review oder dem Economic Journal. Durch Kyklos werden einzig die Daten für die Schweiz und Deutschland stärker beeinflusst, nämlich um 18 bzw. 31 Prozent angehoben. 


\section{Reiner Eichenberger, Ursina Meier und Rolf Arpagaus}

(siehe etwa Kolm 1988). In ihrer einfachen Version ist die SprachbarrierenErklärung aber kaum überzeugend. Zum einen können Texte mit Hilfe von professionellen Übersetzern oder auch sprachkundigen universitären Hilfskräften in publizierbares Englisch gebracht werden. Zum andern vermag diese These weder die Unterschiede zwischen den kontinental-europäischen Ländern zu erklären, noch den Umstand, dass viele Europäer, die an amerikanische Universitäten wechseln, wissenschaftliche Lorbeeren erreichen, lange bevor sie perfekt Amerikanisch schreiben.

Tatsächlich können aber Sprachunterschiede und Landesgrenzen auf zweierlei Weisen eine Rolle spielen, wenn auch nicht direkt auf dem Publikationsmarkt:

Wie Frey und Eichenberger (1992, 1993) zeigen, unterscheiden sich die akademischen Märkte in kleinen Ländern systematisch von denjenigen in grossen Ländern. So dienen im politisch-ökonomischen Prozess regelmässig Unterschiede in der Landessprache zur Abschottung akademischer Märkte, etwa wenn verlangt wird, dass die Lehre in der Landessprache zu halten ist. Dies verschafft natürlich einheimischen Akademikern einen Vorsprung, wodurch der Wettbewerbsdruck vor allem in kleinen Ländern bzw. Sprachräumen gemindert wird.

Zum anderen sind akademische Ökonomen - wenn sie wissenschaftlich aktiv sein wollen - auf eine „Zuhörerschaft" einer gewissen Grösse angewiesen. So gibt es z.B. in vielen kleinen Ländern keine (oder nur einzelne) wissenschaftliche Publikationsorgane und oft kein genügend grosses wissenschaftliches Publikum, um darauf eine akademische Karriere aufzubauen. Ökonomen aus kleinen Ländern haben deshalb vermehrt Anreize, sich entweder in nicht-akademischen Bereichen zu betätigen (z.B. selbst aktiv in die Politik zu gehen), oder aber $\mathrm{zu}$ versuchen, auf dem internationalen akademischen Markt erfolgreich zu sein. Selbstverständlich beeinflusst diese Entscheidung die Themenwahl und die Forschungsmethode. In grösseren Ländern hingegen wie in Italien, Frankreich und auch in Deutschland gibt es einen wissenschaftlichen Markt für Ökonomen, der genügend gross ist, um darauf wissenschaftliche Karrieren aufzubauen. Da diese Märkte aber kleiner sind als der angelsächsische Markt, sind sie weniger durch den anonymen wissenschaftlichen Wettbewerb bestimmt, u.a. weil die Zahl konkurrierender, spezialisierter Fachzeitschriften wesentlich kleiner ist. Deshalb sind die Karrieremöglichkeiten weniger durch die erfolgreiche Publikation von Aufsätzen bestimmt, sondern stärker als im internationalen Markt durch gute persönliche Beziehungen $\mathrm{zu}$ anderen Forschern und $\mathrm{zu}$ Institutionen, nicht zuletzt der Verwaltung und der Regierung. Dies wiederum beeinflusst die Publikationssprache, die Forschungsstrategie und die Auswahl der Forschungsthemen, die sich vermehrt auf lokale und nationale Fragestellungen beziehen müssen (siehe dazu auch Frey und Eichenberger, 1997). Dadurch entsteht Forschung, die auf dem internationalen Markt nicht oder nur schwer unterzubringen ist: Sie ist weder in angesehenen internationalen Zeitschriften publizierbar, noch wird sie in diesen häufig zitiert. 


\section{Ökonomen, Publikationen und Zitationen}

Als Folge davon entstehen in den grossen europäischen Ländern wissenschaftliche Märkte, die zu einem gewissen Grad vom internationalen Markt abgeschottet sind und sich unabhängig entwickeln. Dies führt auch dazu, dass Forscher aus grossen Ländern weniger in internationalen Fachvereinigungen vertreten sind, diejenigen aus kleinen Ländern hingegen einen weit überproportionalen Anteil der Mitglieder solcher Organisationen stellen. Dies trifft auch auf die European Economic Association zu. Während im August 1995163 Mitglieder in Grossbritannien Wohnsitz hatten, waren die Ökonomen aus den anderen grossen europäischen Ländern ähnlich stark vertreten: 122 kamen aus Frankreich, 147 aus Italien und immerhin 271 aus Deutschland. Demgegenüber waren die Wissenschaftler aus den kleineren Ländern weit überproportional vertreten: 131 kamen aus Belgien, 118 aus den Niederlanden, 88 aus Schweden, 78 aus Norwegen, 68 aus der Schweiz, 57 aus Österreich, 53 aus Finnland, 25 aus Dänemark und immerhin je 7 aus Luxemburg und Island (vgl. dazu auch Kirman und Dahl, 1994).

Der eben beschriebene Mechanismus ist nicht auf die Wirtschaftswissenschaften beschränkt. Auch in anderen Wissenschaften und akademischen Märkten existieren solche Eintrittsbarrieren. Besonders typisch dafür dürfte die Rechtswissenschaft sein, die sich - noch ausgeprägter als die Ökonomik - mit nationalen und lokalen, zwischen den Ländern stark differierenden Institutionen befasst. Dadurch wird die Mobilität der Forscher zusätzlich eingeschränkt. In den Naturwissenschaften andererseits dürften die Märkte wesentlich offener sein, weil sich ein grosser Teil der Forschung auf Fragen bezieht, die sich überall auf der Welt in gleicher oder vergleichbarer Weise stellen, und deshalb die Forscher automatisch dem internationalen Wettbewerb ausgesetzt sind. Wie stark hingegen die Marktabschottungen in den anderen Sozialwissenschaften sein können, belegt die sehr sorgfältige Untersuchung über die Psychologie im deutschen Sprachraum von Keul, Gigerenzer und Stroebe (1993). Sie finden eine negative Korrelation der Zitierungen der Werke führender deutschsprachiger Psychologen in deutschsprachigen und englischsprachigen Zeitschriften: je besser also ein deutschsprachiger Autor im internationalen Markt aufgenommen wird, desto weniger wird von ihm im deutschen Markt Notiz genommen. Ob die Märkte für wirtschaftswissenschaftliche Forschung so weitgehend abgeschottet sind, kann leider mit unserem Zahlenmaterial nicht beantwortet werden, ist aber aufgrund der oben angeführten Überlegungen nicht auszuschliessen.

\section{$\overline{\text { V.Schlussfolgerungen }}$}

Die hier vorgestellten Daten zeigen, dass die Dominanz der britischen Ökonomen in Europa schwächer ausgeprägt ist, als oft vermutet wird. Die Briten publizieren zwar weit mehr in international orientierten Fachzeitschriften als ihre deutschen, italienischen und französischen Kollegen, und ihre Aufsätze werden überdies häufiger zitiert. Verglichen mit den 


\section{Reiner Eichenberger, Ursina Meier und Rolf Arpagaus}

Ökonomen aus kleinen westeuropäischen Ländern ist ihr Vorsprung jedoch bedeutend kleiner, wenn die Daten um die Bevölkerungsgrösse korrigiert werden. Die Forscher aus diesen kleinen Ländern sind stärker auf den internationalen Markt ausgerichtet als diejenigen aus den grossen europäischen Ländern. Die Märkte der grossen Länder sind in gewisser Weise geschlossener, und in ihnen können Forscher wissenschaftliche Karrieren aufbauen, ohne sich stark auf den internationalen Markt zu konzentrieren. Diese Erkenntnis ist für weitere Vergleiche der Forschungsaktivität in den verschiedenen Ländern wichtig: Offensichtlich werden die hier verwendeten und international gebräuchlichen Masse zur Outputmessung von Wissenschaftlern den Ökonomen aus den grossen Ländern mit einem selbsttragenden Wissenschaftsmarkt nicht vollauf gerecht. Mehr als anderswo scheinen für die in diesen Ländern lebenden Ökonomen neben den Publikationen in internationalen und nationalen Fachzeitschriften auch andere Leistungsaspekte (wie eine erfolgreiche politische Karriere oder Einfluss durch Beratungsaufträge) wichtig zu sein. Welches Mass aber die Leistung und den Einfluss dieser Ökonomen adäquater erfassen könnte, muss die weitere Forschung beantworten.

\section{$\underline{\underline{\text { Literaturverzeichnis }}}$}

Blaug, M. (Hrsg.), (1986), Who's Who in Economics. A Biographical Dictionary of Major Economists, 1700-1986. Wheatsheaf, Brighton, 2. Aufl.

Blaug, M. (Hrsg.), (1999), Who's Who in Economics. A Biographical Dictionary of Major Economists, 1700-1999. Elgar, Cheltenham, 3. Aufl.

Bommer, R. und H.W. Ursprung (1998), Spieglein, Spieglein an der Wand. Eine publikationsanalytische Erfassung der Forschungsleistungen volkswirtschaftlicher Fachbereiche in Deutschland, Österreich und der Schweiz, Zeitschrift für Wirtschaftsund Sozialwissenschaften 118, 1-28.

Burton, M.P. und E. Phimister (1995), Core Journals: A Reappraisal of the Diamond list, Economic Journal 105, 361-373.

Conroy, M.E., R. Dusansky, D. Drukker und A. Kildegaard (1995), The Productivity of Economics Departments in the U.S.: Publications in the Core Journals, Journal of Economic Literature 33, 1966-1971.

Dusansky, R. und C.J. Vernon (1998), Rankings of U.S. Economics Departments, Journal of Economic Perspectives 12 (1, Winter), 157-170.

Figlio, D. (1994), Trends in the Publication of Empirical Economics, Journal of Economic Perspectives 8 (3, Summer), 179-187.

Frey, B.S. und R. Eichenberger (1992), Economics and Economists: A European View, American Economic Review 82, 216-220.

Frey, B.S. und R. Eichenberger (1993), American and European Economics and Economists: An Analytical Comparison, Journal of Economic Perspectives 7 (4, Fall), 185-193.

Frey, B.S. und R. Eichenberger (1997), Economists: First Semester, High Flyers and UFOs, in: P.A.G. van Bergeijk, A.L. Bovenberg, E.C. van Damme und J. van Sinderen (Hrsg.), Economic Science and Practice. The Roles of Academic Economists and Policymakers. Elgar, Cheltenham, 15-48. 


\section{Ökonomen, Publikationen und Zitationen}

Frey, B.S. und W.W. Pommerehne (1988), The American Domination Among Eminent Economists, Scientometrics 14, 97-110.

Hodgson, G.M. und H. Rothman (1999), The Editors and Authors of Economics Journals: A Case of Institutional Oligopoly? Economic Journal 109, F165-F186.

Kalaitzidakis, P., T.P. Mamuneas und T. Stengos (1999), European Economics: An analysis based on publications in the core journals, European Economic Review 43, 1150-1168.

Keul, A.G., G. Gigerenzer und W. Stroebe (1993), Wie International ist die Psychologie in Deutschland, Österreich und der Schweiz? Eine SSCI-Analyse, Psychologische Rundschau 44, 259-269.

Kirman, A. und M. Dahl (1994), Economic Research in Europe, European Economic Review $38,505-522$.

Kolm, S.-Ch. (1988), Economics in Europe and in the U.S., European Economic Review 32, 207-212.

Laband, D.N. (1985), An Evaluation of 50 „Ranked“ Economics Departments - By Quantity and Quality of Faculty Publications and Graduate Student Placements and Research Success, Southern Economic Journal 52, 216-240.

Laband, D.N. und M.J. Piette (1994), The Relative Impact of Economics Journals: 19701990, Journal of Economic Literature 32, 640-666.

Liebowitz, St.J. und J.P. Palmer (1984), Assessing the Relative Impacts of Economics Journals, Journal of Economic Literature 22, 77-88.

Meier, U.B. (1994), Wissenschaftliche Publikationsaktivitäten an Hochschulen - ein Vergleich der volkswirtschaftlichen Abteilungen deutschschweizerischer Hochschulen, Schweizerische Zeitschrift für Volkswirtschaft und Statistik 130, 207-232.

Pommerehne, W.W. und B.S. Frey (1988), Die Geltung deutschsprachiger Ökonomen in der Welt, Jahrbücher für Nationalökonomie und Statistik 204/5, 406-422.

Portes, R. (1987), Economics in Europe, European Economic Review 31, 1329-1340.

Social Sciences Citation Index (1992), Social Sciences Citation Index. 1986-90 Five Year Cumulation. Institute for Scientific Information, Philadelphia.

Social Sciences Citation Index (1993-96), Social Sciences Citation Index. Institute for Scientific Information (verschiedene Jahrgänge), Philadelphia.

Steinmann, H., Th. Olbrich und H. Riedl (1998), Die deutschsprachige Forschung zur Unternehmensstrategie in der Selbsteinschätzung von Hochschulen, in: G. Handlbauer, K. Matzler, E. Sauerwein und M. Stumpf (Hrsg.), Perspektiven im Strategischen Management. de Gruyter, Berlin, 369-385.

Stigler, G.J., St.M. Stigler und C. Friedland (1995), The Journals of Economics, Journal of Political Economy 103, 331-359.

Strate, J., M. Winterhager und R. Sehringer (1991), Der Stand der schweizerischen Grundlagenforschung im internationalen Vergleich, Wissenschaftspolitik (Beiheft 51).

Woolgar, St. (1991), Beyond the Citation Debate: Towards a Sociology of Measurement Technologies and their Use in Science Policy, Science and Public Policy 18, 319-326. 
Abstract: This paper measures the publishing activity and the impact of European economists, 1986-1996. The analysis of the number of publications in selected scholarly journals and the citations shows that, relative to population size, the British economists are in the lead. They publish much more than their German, French and Italian colleagues. At the same time, their work seems also to be cited more often. However, compared to the economists from the small western European countries, the British dominance is much less pronounced. The latter seem to be much more focused on the international academic market than scholars from the large continental countries. 